\title{
Integral Means Inequalities, Convolution, and Univalent Functions
}

\author{
Daniel Girela (iD) and Cristóbal González \\ Análisis Matemático, Universidad de Málaga, Campus de Teatinos, 29071 Málaga, Spain \\ Correspondence should be addressed to Daniel Girela; girela@uma.es
}

Received 19 December 2018; Accepted 5 February 2019; Published 3 March 2019

Academic Editor: Konstantin M. Dyakonov

Copyright (C) 2019 Daniel Girela and Cristóbal González. This is an open access article distributed under the Creative Commons Attribution License, which permits unrestricted use, distribution, and reproduction in any medium, provided the original work is properly cited.

We use the Baernstein star-function to investigate several questions about the integral means of the convolution of two analytic functions in the unit disc. The theory of univalent functions plays a basic role in our work.

\section{Introduction}

Let $\mathbb{D}=\{z \in \mathbb{C}:|z|<1\}$ and $\mathbb{T}=\{z \in \mathbb{C}:|z|=1\}$ denote the open unit disc and the unit circle in the complex plane $\mathbb{C}$. We let also $\mathscr{H}$ ol $(\mathbb{D})$ be the space of all analytic functions in $\mathbb{D}$ endowed with the topology of uniform convergence in compact subsets.

If $0 \leq r<1$ and $f \in \mathscr{H}$ ol $(\mathbb{D})$, we set

$$
M_{p}(r, f)=\left(\int_{-\pi}^{\pi}\left|f\left(r e^{i t}\right)\right|^{p} \frac{d t}{2 \pi}\right)^{1 / p}
$$

$$
\text { if } 0<p<\infty \text {, }
$$

$$
M_{\infty}(r, f)=\sup _{|z|=r}|f(z)| .
$$

For $0<p \leq \infty$, the Hardy space $H^{p}$ consists of those $f \in \mathscr{H}$ ol $(\mathbb{D})$ such that

$$
\|f\|_{H^{p}} \stackrel{\text { def }}{=} \sup _{0 \leq r<1} M_{p}(r, f)<\infty .
$$

We refer to [1] for the theory of $H^{p}$-spaces.
If $f, g \in \mathscr{H} o l(\mathbb{D})$

$$
\begin{aligned}
& f(z)=\sum_{n=0}^{\infty} a_{n} z^{n}, \\
& g(z)=\sum_{n=0}^{\infty} b_{n} z^{n}
\end{aligned}
$$

$$
(z \in \mathbb{D}),
$$

the (Hadamard) convolution $(f \star g)$ of $f$ and $g$ is defined by

$$
(f \star g)(z)=\sum_{n=0}^{\infty} a_{n} b_{n} z^{n}, \quad z \in \mathbb{D} .
$$

We have the following integral representation:

$$
(f \star g)(z)=\frac{1}{2 \pi i} \int_{|\xi|=r} f\left(\frac{z}{\xi}\right) g(\xi) \frac{d \xi}{\xi}, \quad|z|<r<1,
$$

(see $[2$, p. 11]). The convolution operation $\star$ makes $\mathscr{H}$ ol $(\mathbb{D})$ into a commutative complex algebra with an identity

$$
I(z)=\frac{1}{1-z}=\sum_{n=0}^{\infty} z^{n}, \quad z \in \mathbb{D} .
$$


We refer to [2] for the theory of the convolution of analytic functions and its connections with geometric function theory.

Following [3], we shall say that a function $F \in \mathscr{H}$ ol $(\mathbb{D})$ is bound preserving if for every $f \in H^{\infty}$ we have that $f \star F \in$ $H^{\infty}$ and

$$
\|f \star F\|_{H^{\infty}} \leq\|f\|_{H^{\infty}} .
$$

Sheil-Small [3, Theorem 1.3] (see also [2, p. 123]) proved that a function $F \in \mathscr{H}$ ol $(\mathbb{D})$ is bound preserving if and only if there exists a complex Borel measure $\mu$ on $\mathbb{T}$ with $\|\mu\| \leq 1$ such that

$$
F(z)=\int_{\mathbb{T}} \frac{d \mu(\xi)}{1-z \xi}, \quad z \in \mathbb{D} .
$$

The measure $\mu$ is a probability measure if and only if $F$ is convexity preserving; that is, for any $f \in \mathscr{H}$ ol $(\mathbb{D})$ the range of $f \star F$ is contained in the closed convex hull of the range of $f[2$, pp. 123, 124].

It turns out that if $F$ is bound preserving and $1 \leq p \leq \infty$, then for every $f \in H^{p}$ we have that $f \star F \in H^{p}$ and

$$
\|f \star F\|_{H^{p}} \leq\|f\|_{H^{p}} .
$$

Actually, the following stronger result holds.

Theorem 1. Suppose that $f, F \in \mathscr{H}$ ol $(\mathbb{D})$ with $F$ being bound preserving. Then

$$
M_{p}(r, f \star F) \leq M_{p}(r, f), \quad 0<r<1,
$$

whenever $1 \leq p \leq \infty$.

Proof. Since $F$ is bound preserving, there exists a complex Borel measure $\mu$ on $\mathbb{T}$ with $\|\mu\| \leq 1$ such that

$$
F(z)=\int_{\mathbb{I}} \frac{d \mu(\xi)}{1-z \xi}=\sum_{n=0}^{\infty}\left(\int_{\mathbb{T}} \xi^{n} d \mu(\xi)\right) z^{n}, \quad z \in \mathbb{D} .
$$

If $f(z)=\sum_{n=0}^{\infty} a_{n} z^{n}(z \in \mathbb{D})$, we have

$$
\begin{aligned}
(f \star F)(z) & =\sum_{n=0}^{\infty} a_{n}\left(\int_{\mathbb{T}} \xi^{n} d \mu(\xi)\right) z^{n} \\
& =\int_{\mathbb{T}}\left(\sum_{n=0}^{\infty} a_{n} \xi^{n} z^{n}\right) d \mu(\xi) \\
& =\int_{\mathbb{T}} f(\xi z) d \mu(\xi), \quad z \in \mathbb{D} .
\end{aligned}
$$

This immediately yields (10) for $p=\infty$. Now, if $1 \leq p<\infty$, using Minkowski's integral inequality we obtain

$$
\begin{aligned}
& M_{p}(r, f \star F) \\
& \quad=\left[\frac{1}{2 \pi} \int_{-\pi}^{\pi}\left|\int_{\mathbb{T}} f\left(r \xi e^{i \theta}\right) d \mu(\xi)\right|^{p} d \theta\right]^{1 / p} \\
& \quad \leq\left[\frac{1}{2 \pi} \int_{-\pi}^{\pi}\left(\int_{\mathbb{T}}\left|f\left(r \xi e^{i \theta}\right)\right| d|\mu|(\xi)\right)^{p} d \theta\right]^{1 / p}
\end{aligned}
$$

$$
\begin{aligned}
& \leq \int_{\mathbb{T}}\left(\frac{1}{2 \pi} \int_{-\pi}^{\pi}\left|f\left(r \xi e^{i \theta}\right)\right|^{p} d \theta\right)^{1 / p} d|\mu|(\xi) \\
& =\int_{\mathbb{T}} M_{p}(r, f) d|\mu|(\xi) \leq M_{p}(r, f) .
\end{aligned}
$$

\section{Star-Type Inequalities}

The main purpose of this article is studying the possibility of extending Theorem 1 to cover other integral means, at least for some special classes of functions. In order to do so, we shall use the method of the star-function introduced by A. Baernstein $[4,5]$.

If $u$ is a subharmonic function in $\mathbb{D} \backslash\{0\}$, the function $u^{*}$ is defined by

$$
u^{*}\left(r e^{i \theta}\right)=\sup _{|E|=2 \theta} \int_{E} u\left(r e^{i t}\right) d t
$$

$$
0<r<1,0 \leq \theta \leq \pi
$$

where $|E|$ denotes the Lebesgue measure of the set $E$. The basic properties of the star-function which make it useful to solve extremal problems are the following [5].

(i) If $u$ is a subharmonic function in $\mathbb{D} \backslash\{0\}$, then the function $u^{*}$ is subharmonic in $\mathbb{D}^{+}=\left\{z=r e^{i \theta}: 0<\right.$ $r<1,0<\theta<\pi\}$ and continuous in $\left\{z=r e^{i \theta}: 0<\right.$ $r<1,0 \leq \theta \leq \pi\}$.

(ii) If $v$ is harmonic in $\mathbb{D} \backslash\{0\}$, and it is a symmetric decreasing function on each of the circles $\{|z|=r\}$ $(0<r<1)$, then $v^{*}$ is harmonic in $\mathbb{D}^{+}$and; in fact, $v^{*}\left(r e^{i \theta}\right)=\int_{-\theta}^{\theta} v\left(r e^{i t}\right) d t$.

The relevance of the star-function to obtain integral means estimates comes from the following result.

Proposition A (see [5]). Let $u$ and $v$ be two subharmonic functions in $\mathbb{D}$. Then the following two conditions are equivalent:

(i) $u^{*} \leq v^{*}$ in $\mathbb{D}^{+}$.

(ii) For every convex and increasing function $\Phi: \mathbb{R} \rightarrow \mathbb{R}$, we have that

$\int_{-\pi}^{\pi} \Phi\left(u\left(r e^{i \theta}\right)\right) d \theta \leq \int_{-\pi}^{\pi} \Phi\left(v\left(r e^{i \theta}\right)\right) d \theta$,

$$
0<r<1 \text {. }
$$

Proposition A yields the following result about analytic functions.

Proposition B. Let $f$ and $g$ be two nonidentically zero analytic functions in $\mathbb{D}$. Then the following conditions are equivalent:

(i) $(\log |f|)^{*} \leq(\log |g|)^{*}$ in $\mathbb{D}^{+}$. 
(ii) For every convex and increasing function $\Phi: \mathbb{R} \rightarrow \mathbb{R}$, we have that

$$
\begin{array}{r}
\int_{-\pi}^{\pi} \Phi\left(\log \left|f\left(r e^{i \theta}\right)\right|\right) d \theta \leq \int_{-\pi}^{\pi} \Phi\left(\log \left|g\left(r e^{i \theta}\right)\right|\right) d \theta \\
0<r<1 .
\end{array}
$$

Since for any $p>0$ the function $\Phi$ defined by $\Phi(x)=$ $\exp (p x)(x \in \mathbb{R})$ is convex and increasing, we deduce that if $f$ and $g$ are as in Proposition B and $(\log |f|)^{*} \leq(\log |g|)^{*}$ in $\mathbb{D}^{+}$, then

$$
M_{p}(r, f) \leq M_{p}(r, g), \quad 0<r<1
$$

for all $p>0$.

The main achievement in the use of the star-function by A. Baernstein in [5] was the proof that the Koebe function $k(z)=z /(1-z)^{2}(z \in \mathbb{D})$ is extremal for the integral means of functions in the class $S$ of univalent functions (see $[1,6]$ for the notation and results regarding univalent functions). Namely, Baernstein proved that if $f \in S$ then

$$
( \pm \log |f|)^{*} \leq( \pm \log |k|)^{*}
$$

and, hence,

$$
\int_{-\pi}^{\pi}\left|f\left(r e^{i \theta}\right)\right|^{p} d \theta \leq \int_{-\pi}^{\pi}\left|k\left(r e^{i \theta}\right)\right|^{p} d \theta, \quad 0<r<1,
$$

for all $p \in \mathbb{R}$. In particular, we have that if $f \in S$ and $0<p \leq$ $\infty$, then

$$
M_{p}(r, f) \leq M_{p}(r, k), \quad 0<r<1
$$

Subsequently the star-function has been used in a good number of papers to obtain bounds on the integral means of distinct classes of analytic functions (see, e.g., [7-12]).

Coming back to convolution, the following questions arise in a natural way.

Question 1. Let $f, g, F, G$ be analytic functions in $\mathbb{D}$ with $|F|$ and $|G|$ being symmetric decreasing on each of the circles $\{|z|=r\}$ and suppose that

$$
\begin{aligned}
(\log |f|)^{*} & \leq(\log |F|)^{*} \\
\text { and }(\log |g|)^{*} & \leq(\log |G|)^{*} .
\end{aligned}
$$

Does it follow that $(\log |f \star g|)^{*} \leq(\log |F \star G|)^{*}$ ?

Question 2. Let $F$ and $f$ be two analytic functions in $\mathbb{D}$ and suppose that $F$ is bound preserving. Can we assert that $(\log |f \star F|)^{*} \leq(\log |f|)^{*}$ ?

We shall show that the answer to these two questions is negative. Regarding Question 1 we have the following result.

Theorem 3. There exist two functions $F_{1}, F_{2} \in \mathscr{H}$ ol $(\mathbb{D})$ with

$$
\left(\log \left|F_{j}\right|\right)^{*} \leq(\log |I|)^{*}, \quad \text { for } j=1,2 \text {, }
$$

and such that

$$
\begin{aligned}
& \text { the inequality }\left(\log \left|F_{1} \star F_{2}\right|\right)^{*} \\
& \leq(\log |I \star I|)^{*} \text { does not hold. }
\end{aligned}
$$

Here, I is the identity element of the convolution defined in (6); that is, $I(z)=1 /(1-z)(z \in \mathbb{D})$. Hence $I \star I=I$.

Proof. Let $h$ be an odd function in the class $S$ with Taylor expansion

$$
h(z)=z+a_{3} z^{3}+a_{5} z^{5}+\ldots
$$

with $\left|a_{5}\right|>1$. The existence of such $h$ was proved by Fekete and Szegö (see [13, p. 104]). Set also

$$
h_{1}(z)=\frac{h(z)}{z}=1+a_{3} z^{2}+a_{5} z^{4}+\ldots, \quad z \in \mathbb{D} .
$$

It is well known that there exists a function $H \in S$ such that $h(z)=\sqrt{H\left(z^{2}\right)}\left(\right.$ see $[13$, p. 64] $)$. Set $k_{2}(z)=\sqrt{k\left(z^{2}\right)}=z /(1-$ $\left.z^{2}\right)$ and $J(z)=k_{2}(z) / z=1 /\left(1-z^{2}\right)(z \in \mathbb{D})$. By Baernstein's theorem we have $(\log |H|)^{*} \leq(\log |k|)^{*}$, a fact which easily implies that $\left(\log \left|h_{1}\right|\right)^{*} \leq(\log |J|)^{*}$. Now, it is clear that $J$ is subordinate to $I$ and then, using [8, Lemma 2], we see that $(\log |J|)^{*} \leq(\log |I|)^{*}$. Thus it follows that

$$
\left(\log \left|h_{1}\right|\right)^{*} \leq(\log |I|)^{*} .
$$

For $n=1,2,3, \ldots$, we define $f_{n}$ inductively as follows:

$$
\begin{aligned}
f_{1} & =h_{1} \\
\text { and } f_{n} & =f_{n-1} \star f_{1}, \quad \text { for } n \geq 2 .
\end{aligned}
$$

In other words, $f_{n}=\overbrace{h_{1} \star \cdots \star h_{1}}^{(n)}$. Clearly, (25) yields

$$
f_{n}(z)=1+a_{3}^{n} z^{2}+a_{5}^{n} z^{4}+\ldots
$$

Since $\left|a_{5}\right|>1$, it follows that $\left|a_{5}^{n}\right| \longrightarrow \infty$, as $n \longrightarrow \infty$. This is equivalent to saying that

$$
\left|f_{n}^{(4)}(0)\right| \longrightarrow \infty, \quad \text { as } n \longrightarrow \infty
$$

Then it follows that the family $\left\{f_{n}^{(4)}: n=1,2,3, \ldots\right\}$ is not a locally bounded family of holomorphic functions in $\mathbb{D}$. Using $[14$, Theorem 16, p. 225$]$ we see that the same is true for the family $\left\{f_{n}: n=1,2,3, \ldots\right\}$. Take $p \in(0,1)$, then $I \in H^{p}$. Since a bounded subset of $H^{p}$ is a locally bounded family [1, p. 36], it follows that

$$
\sup _{n \geq 1}\left\|f_{n}\right\|_{H^{p}}=\infty .
$$

Now, (30) implies that $\left\|f_{n}\right\|_{H^{p}}>\|I\|_{H^{p}}$ for some $n$. Using Proposition $\mathrm{B}$, we see that this implies that

$$
\begin{aligned}
& \text { the inequality }\left(\log \left|f_{n}\right|\right)^{*} \\
& \leq(\log |I|)^{*} \text { is not true for some } n .
\end{aligned}
$$

Let $N$ be the smallest of all such $n$. Using (26) and the fact that $f_{1}=h_{1}$, it follows that $N>1$. 
Then it is clear that (23) holds with $F_{1}=f_{1}, F_{2}=f_{N-1}$.

We have the following result regarding Question 2.

Theorem 4. There exist $f, F$ analytic and univalent in $\mathbb{D}$ such that $F$ is convexity preserving and with the property that the inequality $(\log |f \star F|)^{*} \leq(\log |f|)^{*}$ does not hold.

The following lemma will be used in the proof of Theorem 4.

Lemma 5. Let $f, F \in \mathscr{H}$ ol $((D)$ and suppose that $F(0)=1, F$ is convexity preserving, and $f$ and $f \star F$ are zero-free in $\mathbb{D}$ and satisfy the inequality $(\log |f \star F|)^{*} \leq(\log |f|)^{*}$. Then we also have that

$$
\left(\log \left|\frac{1}{f \star F}\right|\right)^{*} \leq\left(\log \left|\frac{1}{f}\right|\right)^{*} .
$$

Proof. Set $u=\log |f \star F|, v=\log |f|$. Then $u$ and $v$ are harmonic in $\mathbb{D}, u(0)=v(0)$, and $u^{*} \leq v^{*}$. Then it follows that, for $0<r<1$ and $0 \leq \theta \leq \pi$,

$$
\begin{aligned}
& (-u)^{*}\left(r e^{i \theta}\right)=\sup _{|E|=2 \theta} \int_{E}-u\left(r e^{i t}\right) d t \\
& =\sup _{|E|=2 \theta}\left(-\int_{-\pi}^{\pi} u\left(r e^{i t}\right) d t+\int_{[-\pi, \pi] \backslash E} u\left(r e^{i t}\right) d t\right) \\
& =-2 \pi u(0)+u^{*}\left(r e^{i(\pi-\theta)}\right) \\
& =-2 \pi v(0)+u^{*}\left(r e^{i(\pi-\theta)}\right) \\
& \leq-2 \pi v(0)+v^{*}\left(r e^{i(\pi-\theta)}\right)=(-v)^{*}\left(r e^{i \theta}\right) .
\end{aligned}
$$

Hence, we have proved that $(-u)^{*} \leq(-v)^{*}$ which is equivalent to (32).

Proof of Theorem 4. Set

$$
\begin{aligned}
& f(z)=\frac{1}{(1-z)^{2}}=\sum_{n=0}^{\infty}(n+1) z^{n}, \\
& F(z)=1-\frac{1}{2} z,
\end{aligned}
$$

$$
z \in \mathbb{D} \text {. }
$$

Clearly, $f$ and $F$ are analytic, univalent, and zero-free in $\mathbb{D}$. Also

$$
(f \star F)(z)=1-z, \quad z \in \mathbb{D} .
$$

Hence $f \star F$ is also zero-free in $\mathbb{D}$. Notice that $1 /(f \star F) \notin H^{\infty}$ and $1 / f \in H^{\infty}$. Then it follows that

$$
\begin{aligned}
& \text { the inequality }\left(\log \left|\frac{1}{f \star F}\right|\right)^{*} \\
& \leq\left(\log \left|\frac{1}{f}\right|\right)^{*} \text { does not hold. }
\end{aligned}
$$

Now, it is a simple exercise to check that

$$
F(z)=\frac{1}{2 \pi} \int_{-\pi}^{\pi} \frac{1-\cos \theta}{1-e^{i \theta} z} d \theta
$$

and then it follows that $F$ is convexity preserving. Then, using (36) and Lemma 5, it follows that the inequality $(\log \mid f \star$ $F \mid)^{*} \leq(\log |f|)^{*}$ does not hold, as desired.

We close the paper with a positive result, determining a class of univalent functions $\mathscr{Z}$ such that (10) is true for all $p>0$, whenever $f \in \mathscr{Z}$ and $F$ is convexity preserving.

A domain $D$ in $\mathbb{C}$ is said to be Steiner symmetric if its intersection with each vertical line is either empty, or is the whole line, or is a segment placed symmetrically with respect to the real axis. We let $\mathscr{Z}$ be the class of all functions $f$ which are analytic and univalent in $\mathbb{D}$ with $f(0)=0, f^{\prime}(0)>0$, and whose image is a Steiner symmetric domain. The elements of $\mathscr{Z}$ will be called Steiner symmetric functions. Using arguments similar to those used by Jenkins [15] for circularly symmetric functions, we see that a univalent function $f$ with $f(0)=0$ and $f^{\prime}(0)>0$ is Steiner symmetric if and only if it satisfies the following two conditions: (i) $f$ is typically real and (ii) $\operatorname{Re} f$ is a symmetric decreasing function on each of the circles $\{|z|=r\}(0<r<1)$. Then it follows that if $f \in \mathscr{Z}$, then for every $r \in(0,1)$, the domain $f(\{|z|<r\})$ is a Steiner symmetric domain and, hence, the function $f_{r}$ defined by $f_{r}(z)=$ $f(r z)(z \in \mathbb{D})$ belongs to $\mathscr{Z}$ and it extends to an analytic function in the closed unit disc $\overline{\mathbb{D}}$. Now we can state our last result.

Theorem 6. Suppose that $f \in \mathscr{Z}$ and let $F$ be an analytic function in $\mathbb{D}$ which is convexity preserving. We have, for every $p>0$,

$$
M_{p}(r, f \star F) \leq M_{p}(r, f), \quad 0<r<1 .
$$

Proof. In view of Theorem 1 we only need to prove (38) for $0<p<1$. Let $\mu$ be the probability measure on $\mathbb{T}$ such that $F(z)=\int_{\mathbb{I}}(d \mu(\xi) /(1-z \xi))(z \in \mathbb{D})$. Then we have

$$
(f \star F)(z)=\int_{\mathbb{T}} f(\xi z) d \mu(\xi) .
$$

Since $F$ is convexity preserving, for $0<r<1$, we have that $\left(f_{r} \star F\right)(\overline{\mathbb{D}})$ is contained in the closed convex hull of $f_{r}(\overline{\mathbb{D}})$. This easily yields

$$
\begin{aligned}
\min _{z \in \mathbb{D}} \operatorname{Re} f_{r}(z) & \leq \min _{z \in \mathbb{D}} \operatorname{Re}\left(f_{r} \star F\right)(z), \\
\max _{z \in \mathbb{D}} \operatorname{Re}\left(f_{r} \star F\right)(z) & \leq \max _{z \in \mathbb{D}} \operatorname{Re} f_{r}(z) .
\end{aligned}
$$

By the remarks in the previous paragraph, we find that, for all $r \in(0,1), f_{r}$ belongs to $\mathscr{Z}$ and extends to an analytic function in the closed unit disc $\overline{\mathbb{D}}$. Finally, we claim that

$$
\left(\operatorname{Re}\left(f_{r} \star F\right)\right)^{*} \leq\left(\operatorname{Re} f_{r}\right)^{*}, \quad 0<r<1 .
$$

Once this is proved, using Proposition 6 of [10], we deduce that

$$
\begin{array}{r}
M_{p}(r, f \star F)=\left\|f_{r} \star F\right\|_{H^{p}} \leq\left\|f_{r}\right\|_{H^{p}}=M_{p}(r, f), \\
0<p \leq 2,
\end{array}
$$

finishing our proof. 
So we proceed to prove (41). Fix $r \in(0,1)$ and set $u=$ $\operatorname{Re}\left(f_{r} \star F\right), v=\operatorname{Re} f_{r}$. Using (39), we have, for $0<R<1$ and $0<\theta<\pi$,

$$
\begin{aligned}
u^{*}\left(R e^{i \theta}\right) & =\sup _{|E|=2 \theta} \int_{E} u\left(R e^{i t}\right) d t \\
& =\sup _{|E|=2 \theta} \int_{E} \int_{\mathbb{T}} v\left(R e^{i t} \xi\right) d \mu(\xi) d t \\
& =\sup _{|E|=2 \theta} \int_{\mathbb{T}} \int_{E} v\left(R e^{i t} \xi\right) d t d \mu(\xi) \\
& \leq \int_{\mathbb{T}} v^{*}\left(\operatorname{Re} e^{i \theta}\right) d \mu(\xi)=v^{*}\left(\operatorname{Re} e^{i \theta}\right) .
\end{aligned}
$$

\section{Data Availability}

No data were used to support this study.

\section{Conflicts of Interest}

The authors declare that there are no conflicts of interest regarding the publication of this paper.

\section{Acknowledgments}

This research is supported by a grant from "El Ministerio de Economía y Competitividad”, Spain (MTM2014-52865-P) and by a grant from la Junta de Andalucía (FQM-210). We wish also to dedicate this article to Fernando Pérez González on the occasion of his retirement.

\section{References}

[1] P. L. Duren, Theory of $H^{p}$ Spaces, Academic Press, New YorkLondon, 1970, Reprint: Dover, Mineola-New York, 2000.

[2] S. Ruscheweyh, Convolutions in Geometric Function Theory, Séminaire de Mathématiques Supérieures [Seminar on Higher Mathematics], Fundamental Theories of Physics, Presses de l'Université de Montréal, Montréal, Canada, 1982.

[3] T. Sheil-Small, "On the convolution of analytic functions," Journal für die Reine und Angewandte Mathematik, vol. 258, pp. 137-152, 1973.

[4] A. Baernstein, "Proof of Edrei's spread conjecture," Proceedings of the London Mathematical Society, vol. 26, no. 3, pp. 418-434, 1973.

[5] A. Baernstein, "Integral means, univalent functions and circular symmetrization," Acta Mathematica, vol. 133, pp. 139-169, 1974.

[6] C. Pommerenke, Univalent Functions, Vandenhoeck \& Ruprecht, Göttingen, Germany, 1975.

[7] A. Baernstein, "Some sharp inequalities for conjugate functions," Indiana University Mathematics Journal, vol. 27, no. 5, pp. 833-852, 1978.

[8] Y. J. Leung, "Integral means of the derivatives of some univalent functions," Bulletin of the London Mathematical Society, vol. 11, no. 3, pp. 289-294, 1979.
[9] J. E. Brown, "Derivatives of close-to-convex functions, integral means and bounded mean oscillation," Mathematische Zeitschrift, vol. 178, no. 3, pp. 353-358, 1981.

[10] D. Girela, "Integral means and BMOA-norms of logarithms of univalent functions," Journal of The London Mathematical Society-Second Series, vol. 33, no. 1, pp. 117-132, 1986.

[11] D. Girela, "Integral means, bounded mean oscillation, and Gel'fer functions," Proceedings of the American Mathematical Society, vol. 113, no. 2, pp. 365-370, 1991.

[12] M. Nowak, "Some inequalities for BMOA functions," Complex Variables Theory and Application, vol. 16, no. 2-3, pp. 81-86, 1991.

[13] P. L. Duren, Univalent Functions, Springer-Verlag, New York, NY, USA, 1983.

[14] L. V. Ahlfors, Complex Analysis. An Introduction to the Theory of Analytic Functions of One Complex Variable, International Series in Pure and Applied Mathematics, McGraw-Hill Book Co., New York, NY, USA, 3rd edition, 1978.

[15] J. A. Jenkins, “On circularly symmetric functions," Proceedings of the American Mathematical Society, vol. 6, pp. 620-624, 1955. 


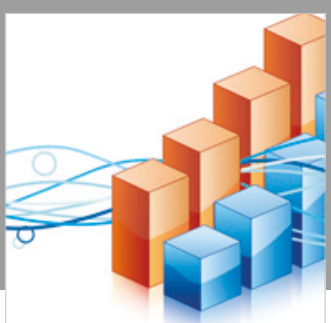

Advances in

Operations Research

\section{-n-m}
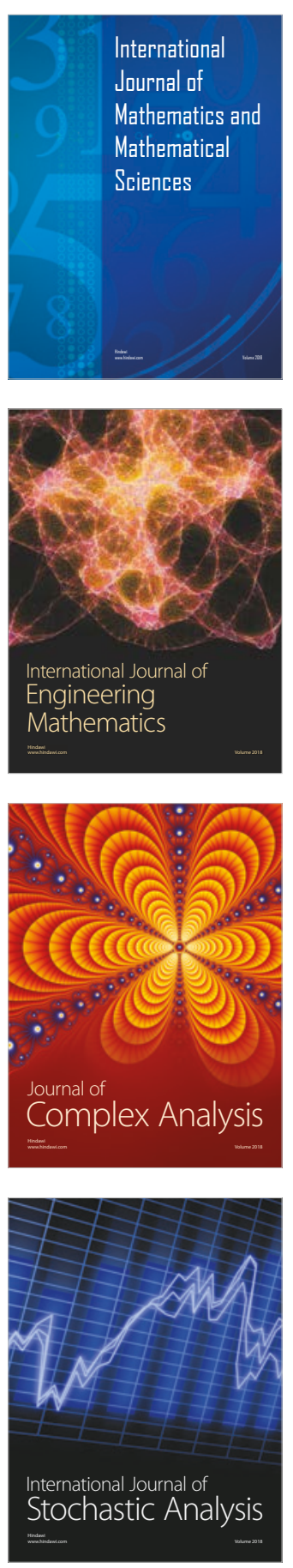
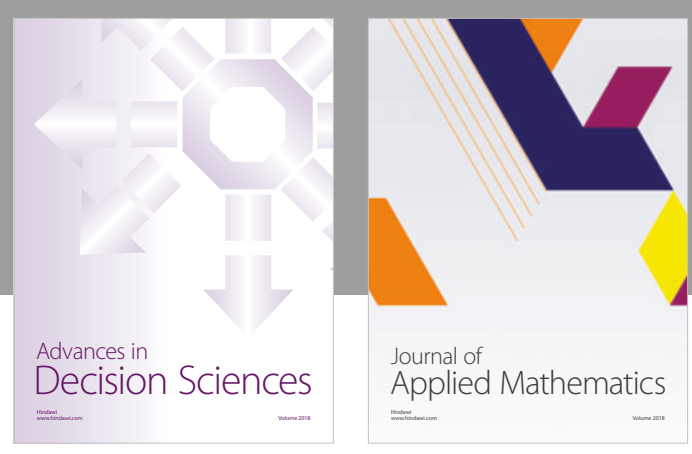

Journal of

Applied Mathematics
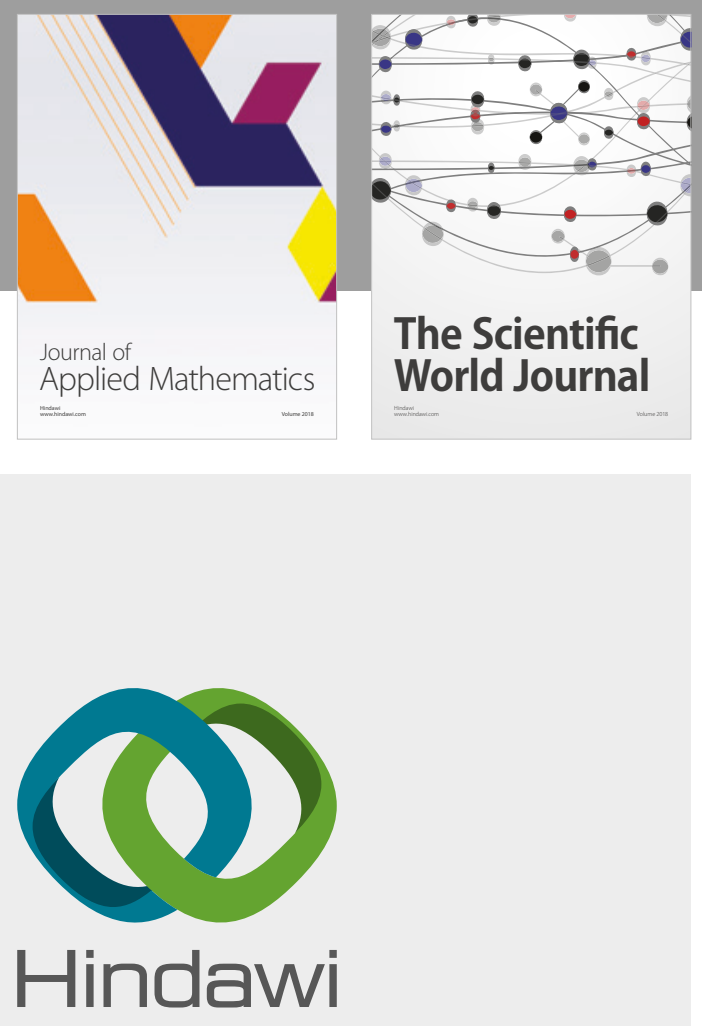

Submit your manuscripts at

www.hindawi.com

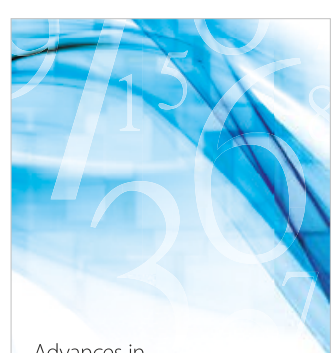

Advances in
Numerical Analysis
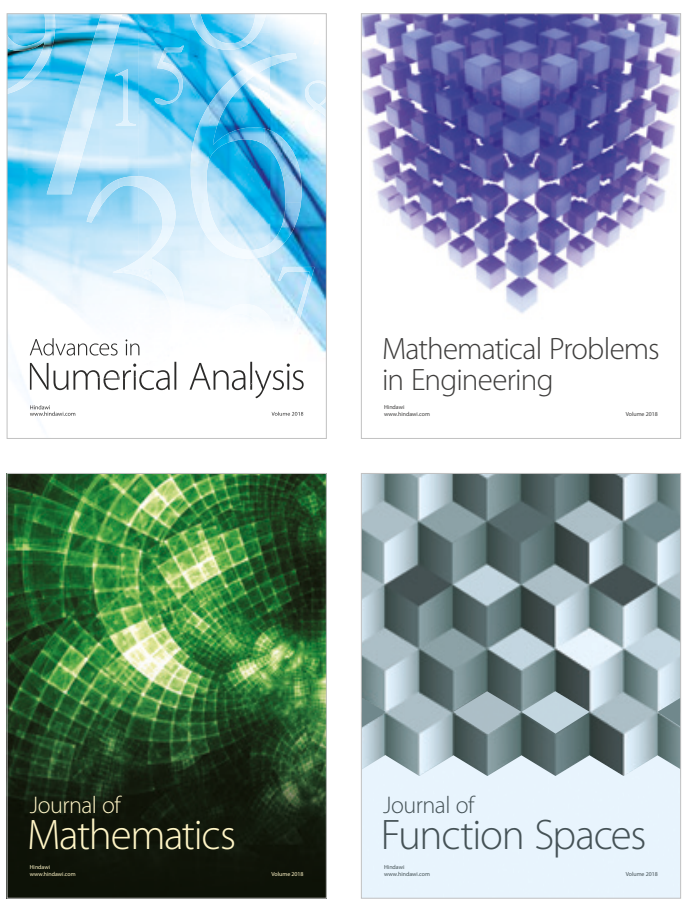

Mathematical Problems in Engineering

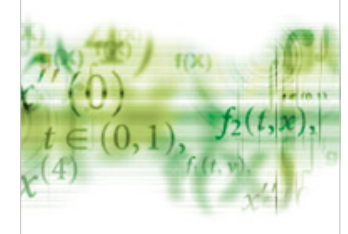

International Journal of

Differential Equations

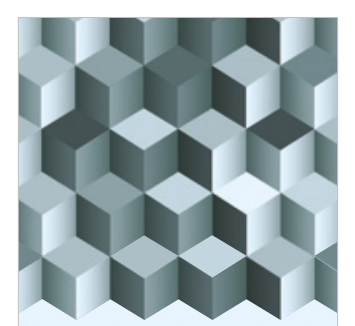

Journal of

Function Spaces

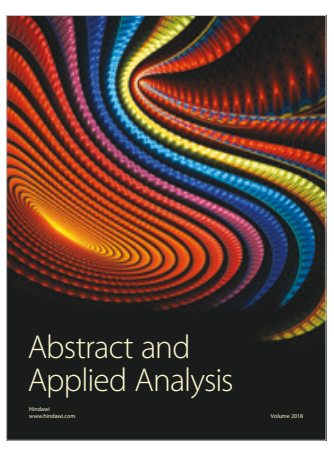

The Scientific

World Journal

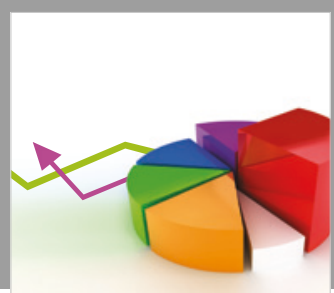

Journal of

Probability and Statistics
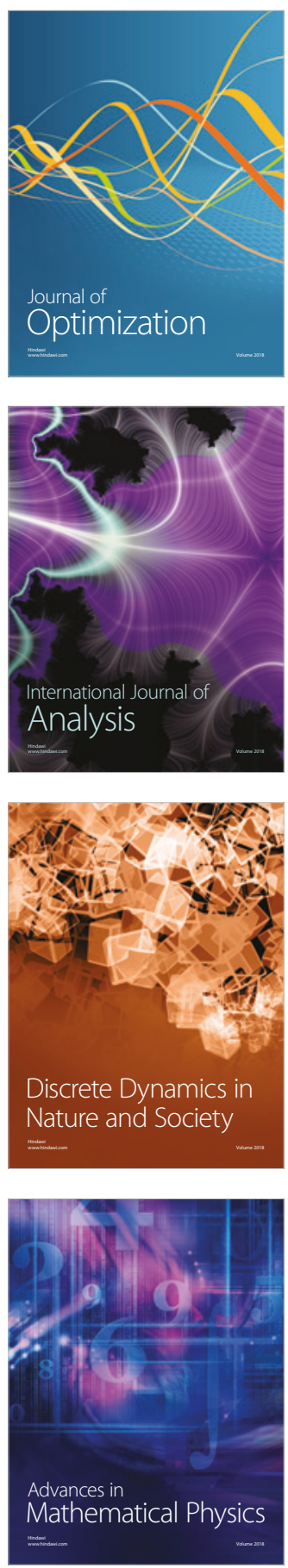